\title{
Níveis de organoclorados em queijos produzidos no Estado do Rio Grande do Sul
}

\author{
Organochlorine levels in the cheese produced in Rio Grande do Sul State
}

\author{
Joice Sifuentes dos Santos ${ }^{1}$ Ana Augusta Odorissi Xavier ${ }^{2}$ Edi Franciele Ries ${ }^{2}$ \\ Ijoni Hilda Costabeber ${ }^{3}$ Tatiana Emanuelli $^{4}$
}

\section{RESUMO}

Os compostos organoclorados são pesticidas que foram usados na agricultura, no controle de vetores biológicos e de ectoparasitas do gado. Atualmente seu uso está proibido no Brasil, exceto para o controle de vetores biológicos em casos de epidemia. No entanto, resíduos desses compostos ainda são encontrados no meio ambiente e nos alimentos. Por esse motivo, avaliou-se a presença de organoclorados em queijos produzidos no Estado do Rio Grande do Sul (Brasil), bem como os níveis desses compostos em queijos dos tipos "colonial" e industrializado. A gordura das amostras foi extraída pela adição de sulfato de sódio seguida por uma lavagem com éter de petróleo. Os pesticidas foram purificados a partir da gordura em uma coluna contendo florisil e eluída com hexano. $O$ extrato obtido foi utilizado para as análises cromatográficas (cromatografia gasosa com captura de elétrons). Os compostos $\propto-H C H, H C B$, lindano, aldrin, o,p-DDD e p,p-DDD foram identificados em $100 \%$ das amostras; o,p-DDE em $88,9 \%$ e o,p-DDT em 94,4\%. p,p-DDD foi o pesticida detectado na maior concentração (11,5ng $\mathrm{g}^{-1}$ de gordura), seguido pelo aldrin (8,7ng $g^{-1}$ de gordura). Os níveis de $\propto-\mathrm{HCH}$, aldrin $e$ DDT total foram maiores que o limite máximo de resíduos em 2, 8 e 1 amostra de queijo, respectivamente. Não foram encontradas diferenças significativas nos níveis de organoclorados entre as amostras de queijo "colonial” $e$ industrializado, ou entre amostras de queijos produzidos em três regiões do Estado.

Palavras-chave: organoclorados, queijo, contaminação.

\section{ABSTRACT}

Organochlorine compounds are pesticides used in agriculture, for the control of biological vectors of diseases and of livestock ectoparasites. Their use in Brazil is now prohibited, except for control of biological vectors in epidemics. However, residues of such compounds can still be found in the environment and in foodstuffs. For this reason, the presence of organochlorine compounds in cheese produced in Rio Grande do Sul state (Brazil), as well as the differences in organochlorine levels between homemade and industrialized cheese were evaluated. Fat extraction from samples was made by grinding with anhydrous sodium sulfate followed by petroleum ether washing. Pesticides were extracted from fat through a florisil column eluted with hexane. The extract was used for the chromatographic analyses (electron capture gas chromatography). $\propto-H C H, H C B$, lindane, aldrin, o,p-DDD, and $p, p-D D D$ were found in $100 \%$ of samples; $p, p-D D E$ in $88.9 \%$, and o,p-DDT in $94.4 \%$. o,p-DDD was the pesticide found at the highest concentration (11.5ng $\mathrm{g}^{-1}$ fat) followed by aldrin (8.7ng $\mathrm{g}^{-1} \mathrm{fat}$ ). The levels of $\propto-\mathrm{HCH}$, aldrin, and total DDT were higher than maximum residue level in 2, 8, and 1 cheese sample, respectively. No significant differences were observed in the levels of organochlorine compounds between homemade and industrialized cheese or among cheese produced in three regions of Rio Grande do Sul.

Key words: organochlorine; cheese; contamination.

\section{INTRODUÇÃO}

Compostos organoclorados tais como hexaclorociclohexanos (HCHs), diclorodifeniltricloroetano (DDT) e seus metabólitos são tóxicos, persistentes e

'Programa de Pós-graduação em Ciência e Tecnologia de Alimentos, Centro de Ciências Rurais (CCR), Universidade Federal de Santa Maria (UFSM), Santa Maria, RS, Brasil. E - mail: joicess@pop.com.br

${ }^{2}$ Curso de Farmácia e Bioquímica, Opção Tecnologia de Alimentos, Centro de Ciências da Saúde (CCS), UFSM, Santa Maria, RS. Brasil.

${ }^{3}$ Departamento de Morfologia, CCS, UFSM, Brasil, Faixa de Camobi Km 9, Campus Universitário, 97105-900, Santa Maria, RS, Brasil. Autor para correspondência. E - mail: ijoni@smail.ufsm.br

${ }^{4}$ Departamento de Tecnologia e Ciência de Alimentos, CCR, UFSM, Faixa de Camobi Km 9, Campus Universitário, 97105-900, Santa Maria, RS, Brasil. E-mail: tati@ccr.ufsm.br 
sofrem bioacumulação (BHATTACHARYA et al., 2003). As propriedades físicas de lipossolubilidade, resistência à metabolização e persistência no ambiente, conferem a estes compostos a capacidade de se depositarem nos tecidos lipídicos dos organismos vivos, sofrendo biomagnificação, alcançando concentrações mais elevadas em animais de níveis tróficos superiores, como os seres humanos. Mesmo que alguns pesticidas não mostrem um efeito imediato in vivo nas concentrações normalmente usadas na agricultura, podem ter um efeito não desejado significativo, a longo prazo, no organismo humano. Eles têm sido associados a vários efeitos crônicos, incluindo imunossupressão, deficiência reprodutiva, alterações endócrinas e neurológicas, carcinogenicidade, mutagenicidade, teratogenicidade, irritação e lesão ocular e cutânea (BENTABOL \& JODRAL, 1995; NASO et al., 2004).

Os pesticidas organoclorados foram utilizados nos Estados Unidos e Europa desde os anos 50 até final da década de 80 , mas a preocupação acerca de seu potencial carcinogênico e prejuízos ambientais acabaram restringindo ou até mesmo banindo seu uso em muitos países. Assim, os níveis desses compostos detectados em alguns alimentos contaminados são resultantes de contaminação indireta de fontes ambientais ou uso impróprio de pesticidas (ABOUARAB, 1997). No Brasil, a Portaria no 329 , de 02 de setembro de 1985 proíbe a comercialização, o uso e a distribuição dos produtos agrotóxicos organoclorados, destinados à agropecuária, em todo território nacional (ANVISA, 1985). O uso desses compostos é permitido apenas em campanhas de saúde pública no combate a vetores de agentes etiológicos de moléstias, quando aplicados pelos órgãos públicos competentes. Como resultado, os níveis de organoclorados nos diversos gêneros alimentícios vêm decrescendo nos últimos anos. Entretanto, estes compostos mantêm relevância econômica em muitos países em desenvolvimento, onde o DDT, por exemplo, é ainda extensamente utilizado no combate à malária (LEMAIRE et al., 2004).

Os resíduos de organoclorados persistentes podem ingressar no organismo humano e animal por diferentes vias, em especial a respiratória e a digestiva (FERNÍCOLA, 1997). O leite é um dos caminhos pelos quais os pesticidas organoclorados são excretados do organismo. Os animais produtores de leite acumulam resíduos desses pesticidas através de alimentos contaminados, pastagem e ar inalado (WALISEWSKI et al., 1997). Quanto à excreção desses resíduos por animais lactantes, esta ocorre principalmente através da gordura do leite (JENSEN, 1983). Segundo esse autor, o conteúdo de lipídios do leite (3-5\%) e o grande fluxo de sangue ao tecido mamário podem conduzir ao acúmulo de altas concentrações desses compostos quando comparado a outros tecidos. Assim, a contaminação, tanto de leite de vaca como de leite humano, pode conduzir a efeitos tóxicos no receptor. Como o leite é matéria-prima de vários produtos, estes também podem ser contaminados, especialmente os mais ricos em gordura, como o queijo.

Os produtos lácteos têm grande importância na dieta humana, especialmente para grupos vulneráveis como bebês, crianças e idosos (OTTOBONI, 1991; BENTABOL \& JODRAL, 1995). FERNÍCOLA (1997) considera que os resíduos de pesticidas organoclorados representam um risco particular como agentes carcinogênicos para as crianças. Isto ocorre devido à maior vulnerabilidade das células a danos no DNA, maior suscetibilidade aos efeitos tóxicos das substâncias químicas e maior ingresso de pesticidas ao organismo infantil quando comparado ao do adulto (OTTOBONI, 1991).

A presença de resíduos de organoclorados em produtos de origem animal, mais concretamente em leite e derivados, é um fato observado por diversos autores (BENTABOL \& JODRAL, 1995; WONG \& LEE, 1997; JOSHI et al., 1998). A maioria dos trabalhos existentes sobre o tema foi realizada na Europa e nos Estados Unidos. A importância de trabalhos desta natureza no Brasil se dá principalmente pelo fato de existirem poucos estudos que tratem sobre a presença de resíduos organoclorados em alimentos no país e, particularmente, no Estado do Rio Grande do Sul. Assim, propôs-se o presente trabalho com o objetivo de avaliar a presença de pesticidas organoclorados em queijos produzidos no Rio Grande do Sul e investigar uma possível diferença nos níveis destes compostos em queijos "coloniais" e industrializados, bem como entre queijos produzidos em diferentes regiões do Estado.

\section{MATERIAL E MÉTODOS}

Foram utilizados 18 queijos, sendo 14 do tipo "colonial", adquiridos em feiras no interior e na capital do Rio Grande do Sul; e quatro do tipo queijo industrializado, adquiridos em supermercados na cidade de Santa Maria - RS, produzidos em diferentes localidades do Estado. Os queijos foram coletados e armazenados $\mathrm{a}-20^{\circ} \mathrm{C}$ até o momento das análises. Denominou-se queijo "colonial" aquele produzido artesanalmente, na maioria dos casos na própria casa do produtor rural, sem obedecer a uma Legislação específica. Como queijo industrializado, foram 
classificados aqueles provenientes de indústrias, produzidos a partir de leite previamente padronizado e seguindo as normas estabelecidas pela Legislação.

Para a determinação da porcentagem de gordura, seguiu-se o método de GERBER (AOAC, 1980), com pequenas modificações. Pesou-se $1 \mathrm{~g}$ de queijo em um béquer, ao qual foi adicionado $10 \mathrm{~mL}$ de ácido sulfúrico $(\mathrm{d}=1,605)$ e levado a banho-maria $\left(\mathrm{T}=60^{\circ} \mathrm{C}\right)$ até completa dissolução. A mistura foi transferida para butirômetro e o béquer lavado três vezes com $3 \mathrm{~mL}$ de ácido sulfúrico. Adicionou-se $1 \mathrm{~mL}$ de álcool isoamílico ao butirômetro, que foi arrolhado e levado ao banhomaria a $65^{\circ} \mathrm{C}$ durante $15 \mathrm{~min}$. Logo após, foi centrifugado por $5 \mathrm{~min}$ para então proceder à leitura.

A gordura foi extraída seguindo a metodologia proposta por WALISZEWSKI et al. (1997), com algumas adaptações. Trituraram-se aproximadamente $20 \mathrm{~g}$ de amostra com sulfato de sódio anidro em gral até obter-se um pó seco e homogêneo. A seguir, o pó foi acondicionado em uma coluna e eluído com éter de petróleo. A fração lipídica foi obtida por evaporação do solvente. A partir desta fração, os compostos foram purificados através de uma coluna cromatográfica contendo florisil. O florisil foi previamente ativado em estufa a $150^{\circ} \mathrm{C}$ por $12 \mathrm{~h}$, e depois parcialmente desativado com $2 \%$ de água ultra purificada. A eluição foi realizada com hexano, e após a evaporação deste solvente realizou-se a reconstituição dos compostos com hexano. Os compostos $\alpha$ hexaclorociclohexano $(\alpha-\mathrm{HCH})$, hexaclorobenzeno (HCB), lindano $(\gamma-\mathrm{HCH})$ e aldrin, e os metabólitos do DDT (p,p-DDE, o,p-DDD, o,p-DDT e p,p-DDD) foram identificados e quantificados com cromatógrafo gasoso AGILENT 6890 com micro detector de captura de elétrons $(\mu \mathrm{ECD})$ de $\mathrm{Ni}^{63}$ e coluna capilar Agilent modelo HP-5 (Crosslinked 5\% PH ME siloxane), de 30m de comprimento, $0,25 \mathrm{~mm}$ de diâmetro interno e $0,25 \mu \mathrm{m}$ de espessura de fase estacionária. As análises cromatográficas foram realizadas segundo as seguintes condições: volume de injeção $1 \mu \mathrm{L}$, temperatura do injetor de $225^{\circ} \mathrm{C}$ e do detector de $300^{\circ} \mathrm{C}$. A temperatura inicial do forno foi de $50^{\circ} \mathrm{C}$ e a final foi de $250^{\circ} \mathrm{C}$. O gás de arraste utilizado foi o nitrogênio. Paralelamente às análises procedeu-se a realização de um branco.

Para avaliar a recuperação do método, efetuou-se a adição de concentrações conhecidas de padrões dos analitos às amostras. A seguir, procedeuse a purificação dos compostos pelo método proposto. As recuperações médias ficaram entre 72,0\% e 120,6\%.

Os dados foram analisados utilizando-se o programa Statistica ${ }^{\circledR}$ 6.0. As diferenças nos níveis de organoclorados entre os dois tipos de queijo foram avaliadas por teste $t$ de Student, enquanto as diferenças entre os queijos produzidos nas 3 regiões do estado foram avaliadas por análise de variância (ANOVA). Para avaliar a relação entre os níveis de organoclorados e o teor de gordura das amostras, aplicou-se o teste de correlação de matrizes. As diferenças foram consideradas significativas quando $\mathrm{P} \leq 0,05$.

\section{RESULTADOS E DISCUSSÃO}

Na tabela 1, puderam-se observar as freqüências e concentrações dos compostos encontradas nas amostras de queijo. Os resultados da corrente pesquisa indicam a presença de organoclorados em queijo "colonial" e industrializado. Nota-se que os compostos analisados estavam

Tabela 1 - Freqüência, concentração média e intervalo de concentração de compostos organoclorados em amostras de queijo (n=18)

\begin{tabular}{lccc}
\hline Composto & Freqüência (\%) & Média \pm DP & Intervalo \\
& & (ng/g de gordura) & $0,1-4,7$ \\
(ng/g de gordura) & $0,7 \pm 1,3$ & $0,4-4,7$ \\
\hline -HCH & 100,0 & $1,8 \pm 1,3$ & $0,1-5,8$ \\
HCB & 100,0 & $0,8 \pm 1,4$ & $2,3-34,6$ \\
Aldrin & 100,0 & $8,7 \pm 8,5$ & $0,0-3,3$ \\
o,p-DDE & 100,0 & $0,5 \pm 1,0$ & $1,6-50,3$ \\
o,p-DDD & 88,9 & $11,5 \pm 12,7$ & $0,1-6,9$ \\
p,p-DDD & 100,0 & $1,3 \pm 1,8$ & $0,0-2,7$ \\
o,p-DDT & 100,0 & $1,0 \pm 0,8$ & $3,9-51,9$ \\
$\sum$ DDT & 94,4 & $14,3 \pm 12,5$ & 3,9 \\
\hline
\end{tabular}

$\mathrm{DP}=$ Desvio padrão.

$\sum$ DDT = Somatório das concentrações dos metabólitos do DDT (o,p-DDE, o,p-DDD, o,p-DDT e p,p-DDD). 
presentes em $100 \%$ das amostras, com exceção do p,pDDE $(88,9 \%)$ e o,p-DDT $(94,4 \%)$. Do total de 18 amostras, $2(11,1 \%), 8(44,4 \%)$ e 1 amostra $(5,5 \%)$ ultrapassaram os níveis permitidos para o $\alpha-\mathrm{HCH}(4 \mathrm{ng}$ $\mathrm{g}^{-1}$ de gordura), aldrin (6 $\left.\mathrm{ng} \mathrm{g}^{-1}\right)$ e DDT total $\left(50 \mathrm{ng} \mathrm{g}^{-1}\right)$, respectivamente. No caso do aldrin, o valor máximo estabelecido na legislação refere-se ao somatório deste composto com o dieldrin. Observa-se, então, que uma parte das amostras analisadas apresentou concentrações menores que os Limites Máximos de Resíduos (LMR) permitidos pela Instrução Normativa $\mathrm{n}$ - 42, do Ministério da Agricultura e do Abastecimento, de 20/12/1999 (BRASIL, 1999). Esta Instrução Normativa estabelece os limites para organoclorados em leite, sendo que estes foram utilizados para comparação devido ao fato de que diversos países, como os da Comunidade Européia (REAL DECRETO 2460/1996), utilizam valores iguais tanto para o leite como para seus derivados. O fato de que $56 \%$ das amostras de queijo apresentaram pelo menos um composto organoclorado em concentrações superiores ao LMR provoca preocupação e aponta para a necessidade de contínuo monitoramento deste alimento, e identificação de possíveis fontes de contaminação.

A concentração média determinada para o DDT total foi de $14,3 \mathrm{ng} \mathrm{g}^{-1}$ de gordura, sendo que o metabólito que mais contribuiu para este somatório foi o o,p-DDD, com uma concentração média de $11,5 \mathrm{ng} \mathrm{g}^{-1}$ de gordura. O DDT apresentou níveis superiores aos demais compostos analisados. Da mesma forma, em estudo realizado na Índia (PANDIT et al., 2002), os níveis de DDT total foram superiores aos demais compostos analisados, o qual foi atribuído às extensivas atividades sanitárias anti-malária. Os níveis do $\Sigma$ DDT encontrados no presente estudo são consideravelmente menores que os relatados por BENTABOL \& JODRAL (1995), porém são maiores do que os descritos por ABOU-ARAB (1997).

BENTABOL \& JODRAL (1995) avaliaram a contaminação por organoclorados em diferentes tipos de queijo na Espanha e detectaram níveis de 46,3ng g-1 de gordura para o $\alpha-\mathrm{HCH} ; 16,7 \mathrm{ng} \mathrm{g}^{-1}$ de gordura para o aldrin; 5,1ng g-1 de gordura para o p,p-DDT; 19,6ng g ${ }^{-1}$ de gordura para o o,p-DDD; 46,7ng g-1 de gordura para o p,p-DDD; e 6,9ng/g de gordura para o p,p-DDE. Comparando esses resultados com os obtidos no presente estudo, observa-se que os queijos espanhóis apresentaram uma contaminação mais elevada. Este fato pode ser atribuído a diferenças nos níveis de contaminação ambiental nas duas regiões, ou a diferença de tempo entre a realização das referidas pesquisas. Considerando que o trabalho espanhol data de 1995, é possível que os compostos organoclorados tenham se degradado e suas concentrações diminuído no decorrer do tempo. KANNAN et al. (2004) sugerem que, após os mesmos terem sido banidos em 1970, suas concentrações têm declinado em vários ecossistemas.

$\mathrm{O} \alpha-\mathrm{HCH}, \mathrm{HCB}$, lindano e aldrin apresentaram concentrações médias de 0,$7 ; 1,8 ; 0,8$ e $8,7 \mathrm{ng} \mathrm{g}^{-1}$ de gordura, respectivamente. Na Grécia, numa investigação de resíduos de organoclorados em queijo, MALLATOU et al. (1997) verificaram as concentrações médias de lindano, HCB, p,p-DDE e aldrin-dieldrin, sendo que as mesmas encontravam-se abaixo dos limites máximos estabelecidos pela União Européia. As concentrações médias de lindano e HCB foram superiores àquelas encontradas no presente estudo, com valores médios de $1,4 \mathrm{ng}^{-1}$ e $5 \mathrm{ng} \mathrm{g}^{-1}$, respectivamente. Da mesma forma, em estudo realizado no Egito (ABOU-ARAB, 1999) para verificar a presença de organoclorados em queijo, a concentração média de lindano foi superior ( $2 \mathrm{ng} \mathrm{g}^{-1}$ de gordura) e HCB não foi detectado.

As amostras foram agrupadas de acordo com o sistema de produção em queijo "colonial" $(n=14)$ e queijo industrializado $(n=4)$. As concentrações médias de pesticidas organoclorados e o desvio padrão estão apresentados na tabela 2. Não houve diferenças significativas $(\mathrm{P}>0,05)$ nos níveis de organoclorados entre os queijos industrializado e "colonial". BENTABOL et al. (1994), em estudo realizado na Espanha, compararam os tipos de queijo "colonial" e industrializado e verificaram que os níveis de HCB no queijo industrializado eram significativamente maiores $(\mathrm{P}<0,001)$.

Tabela 2 - Concentração média de compostos organoclorados segundo o tipo de queijo analisado

\begin{tabular}{|c|c|c|}
\hline Composto & $\begin{array}{l}\text { Queijo colonial"'(n=14) } \\
\text { (ng/g de gordura) }\end{array}$ & $\begin{array}{l}\text { Queijo industrializado } \\
(\mathrm{n}=4)(\mathrm{ng} / \mathrm{g} \text { de gordura })\end{array}$ \\
\hline$\alpha-\mathrm{HCH}$ & $0,9 \pm 1,5$ & $0,4 \pm 0,1$ \\
\hline $\mathrm{HCB}$ & $1,9 \pm 1,4$ & $1,5 \pm 0,8$ \\
\hline Lindano & $0,6 \pm 0,6$ & $1,6 \pm 2,8$ \\
\hline Aldrin & $7,9 \pm 6,0$ & $11,2 \pm 15,7$ \\
\hline o,p-DDE & $0,6 \pm 1,1$ & $0,3 \pm 0,4$ \\
\hline o,p-DDD & $14,0 \pm 13,4$ & $2,6 \pm 0,9$ \\
\hline p,p-DDD & $1,0 \pm 1,3$ & $2,0 \pm 3,3$ \\
\hline o,p-DDT & $1,0 \pm 0,9$ & $0,7 \pm 0,5$ \\
\hline$\sum \mathrm{DDT}$ & $16,7 \pm 13,1$ & $5,7 \pm 2,6$ \\
\hline
\end{tabular}

Os resultados são apresentados como Média \pm Desvio padrão. $\sum \mathrm{DDT}=$ Somatório das concentrações dos metabólitos do DDT (o,p-DDE, o,p-DDD, o,p-DDT e p,p-DDD). 
Tabela 3 - Concentração média de compostos organoclorados segundo a região de origem do queijo analisado

\begin{tabular}{|c|c|c|c|}
\hline Composto & $\begin{array}{c}\text { Serra/Porto Alegre }(n=6) \\
(n g / g \text { de gordura })\end{array}$ & $\begin{array}{l}\text { Sul/ Oeste }(n=8) \\
\text { (ng/g de gordura) }\end{array}$ & $\begin{array}{l}\text { Santa Maria }(\mathrm{n}=4) \\
(\mathrm{ng} / \mathrm{g} \text { de gordura })\end{array}$ \\
\hline$\alpha-\mathrm{HCH}$ & $0,3 \pm 0,1$ & $0,8 \pm 1,4$ & $1,3 \pm 2,2$ \\
\hline HCB & $1,6 \pm 0,6$ & $1,8 \pm 1,5$ & $2,2 \pm 1,6$ \\
\hline Lindano & $1,6 \pm 2,2$ & $0,6 \pm 0,3$ & $0,2 \pm 0,1$ \\
\hline Aldrin & $9,4 \pm 12,5$ & $8,3 \pm 7,0$ & $8,3 \pm 5,9$ \\
\hline $\mathrm{o}, \mathrm{p}-\mathrm{DDE}$ & $0,8 \pm 1,3$ & $0,1 \pm 0,1$ & $1,2 \pm 1,2$ \\
\hline$o, p-D D D$ & $3,5 \pm 2,2$ & $18,4 \pm 15,2$ & $9,8 \pm 10,3$ \\
\hline $\mathrm{p}, \mathrm{p}-\mathrm{DDD}$ & $1,5 \pm 2,7$ & $0,6 \pm 0,7$ & $2,3 \pm 1,8$ \\
\hline o,p-DDT & $0,9 \pm 0,6$ & $1,4 \pm 0,9$ & $0,3 \pm 0,5$ \\
\hline$\sum \mathrm{DDT}$ & $6,6 \pm 2,5$ & $20,4 \pm 15,0$ & $13,5 \pm 11,2$ \\
\hline
\end{tabular}

Os resultados são apresentados como Média \pm Desvio padrão.

$\sum \mathrm{DDT}=$ Somatório das concentrações dos metabólitos do DDT (o,p-DDE, o,p-DDD, o,p-DDT e p,p-DDD).

As concentrações dos organoclorados em estudo segundo a região do Estado em que foram produzidos os queijos podem ser observadas na tabela 3. O lindano e o aldrin foram encontrados em maiores concentrações na Região Serra/Porto Alegre. Na Região Sul/Oeste do Estado, foram detectados os maiores níveis de o,p-DDD e o,p-DDT, bem como de $\Sigma$ DDT. Os compostos $\alpha$-HCH, HCB, o,p-DDE e p,pDDD estavam em maiores níveis na Região de Santa Maria do Estado. Não foram observadas diferenças significativas $(\mathrm{P}>0,05)$ entre os níveis encontrados nas amostras das três regiões em estudo.

$\mathrm{Na}$ tabela 4, estão apresentadas as porcentagens de gordura das amostras de queijo "colonial" e industrializado. A determinação do teor de gordura das amostras de queijo não revelou diferenças significativas $(\mathrm{P}>0,05)$ entre os tipos "colonial" e industrializado. Apesar de se saber que os praguicidas organoclorados depositam-se na porção lipídica dos alimentos, os testes estatísticos não demonstraram

Tabela 4 - Conteúdo lipídico das amostras segundo o tipo de queijo analisado

\begin{tabular}{lcc}
\hline Tipo de queijo & $\begin{array}{l}\text { Valor médio } \\
(\mathrm{g} / 100 \mathrm{~g} \text { de } \\
\text { queijo })\end{array}$ & $\begin{array}{c}\text { Intervalo } \\
(\mathrm{g} / 100 \mathrm{~g} \text { de } \\
\text { queijo })\end{array}$ \\
\hline "Colonial" $(\mathrm{n}=14)$ & 26,6 & $13,2-35,6$ \\
Industrializado $(\mathrm{n}=4)$ & 27,6 & $19,0-32,8$ \\
Total $(\mathrm{n}=18)$ & 26,8 & $13,2-35,6$ \\
\end{tabular}

relação entre o teor de gordura e a presença destes compostos.

\section{CONCLUSÕES}

Considerando os resultados obtidos no presente estudo, pode-se concluir que não existem diferenças significativas entre os níveis de compostos organoclorados detectados nas amostras de queijo "colonial" e industrializado e nas três regiões do Estado avaliadas, e que poucas amostras de queijo obtidas no Estado do Rio Grande do Sul estão acima dos limites máximos de resíduos (LMR) permitidos pela legislação.

\section{AGRADECIMENTOS}

Ao Conselho Nacional de Desenvolvimento Ciêntífico e Tecnológico (CNPq), pelas bolsas concedidas a J.S. dos Santos e a T. Emanuelli (304257/2004-4).

\section{REFERÊNCIAS}

ABOU-ARAB, A.A.K. Effect of Ras cheese manufacturing on the stability of DDT and its metabolites. Food Chemistry, v.59, n.1, p.115-119, 1997.

ABOU-ARAB, A.A.K. Effects of processing and storage of dairy products on lindane residues and metabolites. Food Chemistry, v.64, p.467-473, 1999.

ANVISA, Agência nacional de Vigilância Sanitária, Portaria $n^{\circ}$ 329, 02 de setembro de 1985. Próbe a comercialização, uso e distribuição de produtos agrotóxicos organoclorados destinados à agropecuária, Brasília, 1985. 1p.

AOAC (Association of Official Analytical Chemists). Official methods of analysis. 13.ed. Washington, DC, 1980. 1094p. 
BENTABOL, A. et al. Residual content of hexachlorobenzene in Spanish cheeses. Bulletin Environmental Contamination Toxicology, v.53, p.877-882, 1994.

BENTABOL, A.; JODRAL, M. Occurrence of organochlorine agrochemical residues in Spanish cheeses. Pesticides Science, v.44, p.177-182, 11p, 1995.

BHATTACHARYA, B. et al. Organochlorine pesticide residues in sediments of a tropical mangrove estuary, India: implications for monitoring. Environment International, v.29, p.587$592,2003$.

BRASIL. Ministério da Agricultura e do Abastecimento, Instrução Normativa no 42, de 20 de dezembro de 1999. Plano Nacional de Controle e Resíduos em Produtos de Origem Animal, Brasília, 1999. $11 \mathrm{p}$.

FERNÍCOLA, N. Contaminación de la leche y sus implicaciones en la nutrición humana. Alimentaria, v.97, p.69-72, 1997.

JENSEN, A.A. Chemical contaminants in human milk. Research Veterinary, v.89, p.1-128, 1983.

JOSHI, D.D. et al. Organochlorine pesticide residues in milk of two distinct agro-geoclimatic regions of Uttar Pradesh, India. National Academy Science Letters-India, v.21, n.1-2, p.3032, 1998 .

KANNAN, K. et al. Organochlorine pesticides and polychlorinated biphenyls in California sea lions. Environmental Pollution, v.131, p.425-434, 2004.
LEMAIRE, A.G. et al. Effect of organochlorine pesticides on human androgen receptor activation in vitro. Toxicology and Applied Pharmacology, v.196, p.235-246, 2004.

MALlAtOU, $\mathrm{H}$ et al. Pesticide residues in milk and cheeses from Greece. Science of the Total Environment, v.96, p.111$117,1997$.

NASO, B. et al. Organochlorine pesticides and polychlorinated biphenyls in European roe deer Capreolus capreolus resident in a protected area in Northern Italy. Science of the Total Environment, v.328, n.1-3, p.83-93, 2004.

OTTOBONI, M.A. The dose makes the poison. 2.ed. New York: Van Nostrand Reinhold, 1991. 244p.

PANDIT, G.G. et al. Persistent organochlorine pesticide residues in milk and dairy products in India. Food Additives and Contaminants, v.19, n.2, p.153-157, 2002.

REAL DECRETO 2460/1996 de 2 de diciembre. Fijación de contenidos máximos para los residuos de plaguicidas sobre y en los productos alimenticios de origen animal (BOE n. 300, de 13 de diciembre de 1996). Madri, Espanha, 1996. 3p.

WALISEWSKI, S.M. et al. Organochlorine pesticide residues in cow's milk and butter in Mexico. Science of Total Environmental, v.208, p.127-132, 1997.

WONG, S.K.; LEE, W.O. Survey of organochlorine pesticide residues in milk in Hong Kong (1993-1995). Journal of AOAC International, v.80, n.6, p.1332-1335, 1997. 